\title{
RATIONAL TILINGS BY $n$-DIMENSIONAL CROSSES
}

\begin{abstract}
SÁNDOR SZABÓ
ABSTRaCt. Consider the set of closed unit cubes whose edges are parallel to the coordinate unit vectors $\mathbf{e}_{1}, \ldots, \mathbf{e}_{n}$ and whose centers are $i \mathbf{e}_{i}, 0 \leqslant|i| \leqslant k$, in $n$ dimensional Euclidean space. The union of these cubes is called a cross. This cross consists of $2 k n+1$ cubes: a central cube together with $2 n$ arms of length $k$. A family of translates of a cross whose union is $n$-dimensional Euclidean space and whose interiors are disjoint is a tiling. Denote the set of translation vectors by $\mathbf{L}$. If the vector set $\mathbf{L}$ is a vector lattice, then we say that the tiling is a lattice tiling. If every vector of $\mathbf{L}$ has rational coordinates, then we say that the tiling is a rational tiling, and, similarly, if every vector of $\mathbf{L}$ has integer coordinates, then we say that the tiling is an integer tiling. Is there a noninteger tiling by crosses? In this paper we shall prove that if there is an integer lattice tiling by crosses, if $2 k n+1$ is not a prime, and if $p>k$ for every prime divisor $p$ of $2 k n+1$, then there is a rational noninteger lattice tiling by crosses and there is an integer nonlattice tiling by crosses. We will illustrate this in the case of a cross with arms of length 2 in 55-dimensional Euclidean space. Throughout, the techniques are algebraic.
\end{abstract}

1. Definitions. Let $\mathcal{E}^{n}, R$, and $Z$ be $n$-dimensional Euclidean space, the real number field, and the integer number ring, respectively. Translations of the space belong to the $n$-dimensional vector space $\mathbf{E}^{n}$ over the number field $R$. Let $O$ be a point in $\mathcal{E}^{n}$ and let $\mathbf{e}_{1}, \ldots, \mathbf{e}_{n}$ be an orthonormal basis for $\mathbf{E}^{n}$.

We call the point set

$$
\bigodot_{O}:=\left\{P: \overrightarrow{O P}=c_{1} \mathbf{e}_{1}+\cdots+c_{n} \mathbf{e}_{n} ; 0 \leqslant c_{1} \leqslant 1, \ldots, 0 \leqslant c_{n} \leqslant 1 ; c_{1}, \ldots, c_{n} \in R\right\}
$$

an $n$-dimensional unit cube with preferential vertex $O$.

Let $T$ be a finite subset of $Z$ containing $(0, \ldots, 0),(1,0, \ldots, 0), \ldots,(0, \ldots, 0,1)$ and let $\mathbf{T}$ and $\mathcal{T}_{O}$ be the sets

$$
\mathbf{T}:=\left\{t_{1} \mathbf{e}_{1}+\cdots+t_{n} \mathbf{e}_{n}:\left(t_{1}, \ldots, t_{n}\right) \in T\right\}
$$

and

$$
\mathcal{T}_{O}:=\left\{\Theta_{P}: \overrightarrow{O P} \in \mathbf{T}\right\} .
$$

If $\mathbf{L}$ is a subset of $\mathbf{E}^{n}$, then we use the following notation $\left(\sigma_{O}, \mathbf{L}\right):=\left\{\sigma_{P}: \overrightarrow{O P} \in \mathbf{L}\right\}$. We say that the system $\left(\mathscr{T}_{O}, \mathbf{L}\right)$ is a tiling of the space $\mathcal{E}^{n}$ if the following conditions hold:

(1) $\varepsilon^{n}=\cup\left\{\bigodot_{P}: \bigodot_{P} \in \mathcal{T}_{Q} \in\left(\mathcal{T}_{O}, \mathbf{L}\right)\right\}$, and $e_{Q}$

(2) if int $e_{P} \cap$ int $\bigodot_{Q} \neq \varnothing$ and $\bigodot_{P} \in \sigma_{R} \in\left(\sigma_{O}, \mathbf{L}\right), \bigodot_{Q} \in \sigma_{S} \in\left(\sigma_{O}, \mathbf{L}\right)$, then $\bigodot_{P}=$

Received by the editors December 3, 1981 and, in revised form, May 19, 1982.

1980 Mathematics Subject Classification. Primary 20K01; Secondary 52A45, 05B40, 05B45, 10E30

Key words and phrases. Exact sequence, factorization of abelian groups, lattices, tiling, star body. 
Let $q_{1}, \ldots, q_{n}$ be fixed positive integers. The vector set

$$
\mathbf{X}:=\left\{\left(x_{1} / q_{1}\right) \mathbf{e}_{1}+\cdots+\left(x_{n} / q_{n}\right) \mathbf{e}_{n}: x_{1}, \ldots, x_{n} \in Z\right\}
$$

we call a lattice. Clearly the lattice $\mathbf{X}$ is a free abelian group with $n$ generators. We also call every subgroup of the group $\mathbf{X}$ a lattice. Thus $\mathbf{L}$ is a lattice if $\mathbf{L} \triangleleft \mathbf{X}$. The standard integer lattice is the lattice

$$
\mathbf{M}:=\left\{m_{1} \mathbf{e}_{1}+\cdots+m_{n} \mathbf{e}_{n}: m_{1}, \ldots, m_{n} \in Z\right\} .
$$

The system $\left(\sigma_{0}^{\top}, \mathbf{L}\right)$ is called a lattice system if $\mathbf{L} \triangleleft \mathbf{X}$ and an integer system if $\mathbf{L} \subseteq \mathbf{M}$.

Let $G$ be an abelian group written additively and let $H, A_{1}, \ldots, A_{r}$ be subsets of $G$. If each element $h$ of $H$ is uniquely expressible in the form

$$
h=a_{1}+\cdots+a_{r} ; \quad a_{1} \in A_{1}, \ldots, a_{r} \in A_{r}
$$

(that is, if $h=a_{1}^{\prime}+\cdots+a_{r}^{\prime} ; a_{1}^{\prime} \in A_{1}, \ldots, a_{r}^{\prime} \in A_{r}$, then $a_{1}=a_{1}^{\prime}, \ldots, a_{r}=a_{r}^{\prime}$ ), then we write $H=A_{1}+\cdots+A_{r}$ and speak of a factorization of the subset $H$ by subsets $A_{1}, \ldots, A_{r}$.

Let $T \subseteq Z^{n}$ and $H \subseteq G$. If there is a set of $n$ elements, $g_{1}, \ldots, g_{n}$ in $G$ such that each element of $H$ is uniquely expressible in the form $t_{1} g_{1}+\cdots+t_{n} g_{n},\left(t_{1}, \ldots, t_{n}\right)$ $\in T$, then we write $H=T\left(g_{1}, \ldots, g_{n}\right)$.

For elements $g_{1}, \ldots, g_{n}$ of group $G$ denote by $\left\langle g_{1}, \ldots, g_{n}\right\rangle$ the group generated by $g_{1}, \ldots, g_{n}$, that is, the smallest subgroup of $G$ containing $g_{1}, \ldots, g_{n}$. In other words,

$$
\left\langle g_{1}, \ldots, g_{n}\right\rangle:=\left\{x_{1} g_{1}+\cdots+x_{n} g_{n}: x_{1}, \ldots, x_{n} \in Z\right\} \text {. }
$$

If $G$ is the internal direct product of the cyclic groups $C_{1}, \ldots, C_{r}$ of orders $m_{1}, \ldots, m_{r}$ and generators $g_{1}, \ldots, g_{r}$, respectively, then we will say that $G$ is defined by the equations $m_{1} g_{1}=0, \ldots, m_{r} g_{r}=0$.

2. Tiling space and factoring groups. The next lemma, due to S. K. Stein (see $[8, \mathrm{p}$. 545]), enables us to lift a factoring from a group $G^{\prime}$ to any group $G$ of which $G^{\prime}$ is a homomorphic image.

LeMMA 2.1. Let $G$ and $G^{\prime}$ be abelian groups and let $G^{\prime}=A^{\prime}+B^{\prime}$ be a factorization of $G^{\prime}$ and finally let $\varphi: G \rightarrow G^{\prime}$ be a homomorphism from $G$ onto $G^{\prime}$. Assume that $A$ is a subset of $G$ such that the restriction of $\varphi$ to $A$ is a bijection between $A$ and $A^{\prime}$. Then $G=A+B \varphi^{-1}$ is a factorization of $G$.

THEOREM 2.1. (1) There is a tiling $\left(\widetilde{\sigma}_{O}, \mathbf{L}\right), \mathbf{L} \subseteq \mathbf{X}$, if and only if there is a group $G$, elements $g_{1}, \ldots, g_{n}$ that generate $G$, and positive integers $q_{1}, \ldots, q_{n}$ such that

$$
\begin{aligned}
G= & T\left(q_{1} g_{1}, \ldots, q_{n} g_{n}\right)+\left\{0, g_{1}, 2 g_{1}, \ldots,\left(q_{1}-1\right) g_{1}\right\} \\
& +\cdots+\left\{0, g_{n}, 2 g_{n}, \ldots,\left(q_{n}-1\right) g_{n}\right\}+H,
\end{aligned}
$$

where $H \subseteq G$.

Furthermore,

(2) $\mathbf{L} \triangleleft \mathbf{X}$ if and only if $H \triangleleft G$, and

(3) $\mathbf{L} \subseteq \mathbf{M}$ if and only if $\left(T\left(q_{1} g_{1}, \ldots, q_{n} g_{n}\right)+H\right) \triangleleft G$. 
Proof. (1) Assume that the system $\left(\mathcal{T}_{O}, \mathbf{L}\right), \mathbf{L} \subseteq \mathbf{X}$, is a tiling. So the abelian group $\mathbf{X}$ is expressible in the form

$$
\begin{aligned}
\mathbf{X}= & T\left(\mathbf{e}_{1}, \ldots, \mathbf{e}_{n}\right)+\left\{\mathbf{0},\left(1 / q_{1}\right) \mathbf{e}_{1}, \ldots,\left(\left(q_{1}-1\right) / q_{1}\right) \mathbf{e}_{1}\right\} \\
& +\cdots+\left\{\mathbf{0},\left(1 / q_{n}\right) \mathbf{e}_{n}, \ldots,\left(\left(q_{n}-1\right) / q_{n}\right) \mathbf{e}_{n}\right\}+\mathbf{L} .
\end{aligned}
$$

Assume that the abelian group $G$ is expressible in the form (2.1). Let $\varphi: \mathbf{X} \rightarrow G$ be the mapping defined as

$$
\left(\left(x_{1} / q_{1}\right) \mathbf{e}_{1}+\cdots+\left(x_{n} / q_{n}\right) \mathbf{e}_{n}\right) \varphi=x_{1} g_{1}+\cdots+x_{n} g_{n} ; \quad x_{1}, \ldots, x_{n} \in Z .
$$

Clearly $\varphi$ is a homomorphism from $\mathbf{X}$ onto $G$ and the restriction of $\varphi$ to set

$$
\begin{array}{r}
T\left(\mathbf{e}_{1}, \ldots, \mathbf{e}_{n}\right)+\left\{\mathbf{0},\left(1 / q_{1}\right) \mathbf{e}_{1}, \ldots,\left(\left(q_{1}-1\right) / q_{1}\right) \mathbf{e}_{1}\right\} \\
+\cdots+\left\{\mathbf{0},\left(1 / q_{n}\right) \mathbf{e}_{n}, \ldots,\left(\left(q_{n}-1\right) / q_{n}\right) \mathbf{e}_{n}\right\}
\end{array}
$$

is a bijection between this set and the set

$$
T\left(q_{1} g_{1}, \ldots, q_{n} g_{n}\right)+\left\{0, g_{1}, \ldots,\left(q_{1}-1\right) g_{1}\right\}+\cdots+\left\{0, g_{n}, \ldots,\left(q_{n}-1\right) g_{n}\right\} .
$$

So by Lemma 2.1, $\mathbf{X}$ is expressible in the form (2.2), where $\mathbf{L}=H \varphi^{-1}$. Thus the $\operatorname{system}\left(\sigma_{O}, \mathbf{L}\right)$ is a tiling of $\mathcal{E}^{n}$.

(2) If $\mathbf{L} \triangleleft \mathbf{X}$, then the equations $\mathbf{L} \varphi=H$ and $\mathbf{X} \varphi=G$ imply that $H \triangleleft G$. Conversely, in case $H \triangleleft G$, then, as is well known, $H \varphi^{-1} \triangleleft G \varphi^{-1}$. Thus $\mathbf{L} \triangleleft \mathbf{X}$.

(3) Assume that $\left(T\left(q_{1} g_{1}, \ldots, q_{n} g_{n}\right)+H\right) \triangleleft G$. We shall show that if $\mathbf{I} \in \mathbf{L}$, then $l \in \mathbf{M}$.

Since $\mathbf{l} \in \mathbf{L} \subseteq \mathbf{X}$, we may write

$$
-\mathbf{l}=\left(l_{1} / q_{1}\right) \mathbf{e}_{1}+\cdots+\left(l_{n} / q_{n}\right) \mathbf{e}_{n} ; \quad l_{1}, \ldots, l_{n} \in Z .
$$

Let $u_{i}, r_{i}$ be integers with the property that $l_{i}=u_{i} q_{i}+r_{i}, 0 \leqslant r_{i}<q_{i}$. Thus

$$
-\mathbf{I}=u_{1} \mathbf{e}_{1}+\cdots+u_{n} \mathbf{e}_{n}+\left(r_{1} / q_{1}\right) \mathbf{e}_{1}+\cdots+\left(r_{n} / q_{n}\right) \mathbf{e}_{n} .
$$

Now we apply the homomorphism $\varphi$. Since $l \varphi=: h \in H$,

$$
\left(T\left(q_{1} g_{1}, \ldots, q_{n} g_{n}\right)+H\right) \triangleleft \cdot G ; \quad \mathbf{e}_{1}, \ldots, \mathbf{e}_{n} \in \mathbf{T},
$$

and

$$
\mathbf{T} \varphi=T\left(q_{1} g_{1}, \ldots, q_{n} g_{n}\right),
$$

therefore $\left(u_{1} q_{1} g_{1}+\cdots+u_{n} q_{n} g_{n}+h\right) \in\left(T\left(q_{1} g_{1}, \ldots, q_{n} g_{n}\right)+H\right)$; thus

$$
0=t_{1} q_{1} g_{1}+\cdots+t_{n} q_{n} g_{n}+r_{1} g_{1}+\cdots+r_{n} g_{n}+h^{\prime},
$$

where $h^{\prime} \in H$ and $\left(t_{1}, \ldots, t_{n}\right) \in T$. According to the factorization (2.1),

$$
\left(t_{1}, \ldots, t_{n}\right)=\left(r_{1}, \ldots, r_{n}\right)=(0, \ldots, 0) ; \quad h^{\prime}=0 .
$$

By virtue of (2.3) and (2.4) we have $I \in M$, which was to be proved.

Assume that $\mathbf{L} \subseteq \mathbf{M}$. We shall show that $(\mathbf{T} \varphi+H) \triangleleft G$. Let $\mathbf{t}, \mathbf{t}^{\prime} \in \mathbf{T}$ and $\mathbf{I}$, $\mathbf{I}^{\prime} \in \mathbf{L}$. Since $\left(\mathbf{t}+\mathbf{I}-\left(\mathbf{t}^{\prime}+\mathbf{l}^{\prime}\right)\right) \in \mathbf{M}$ and the system $\left(\widetilde{T}_{O}, \mathbf{L}\right)$ is a tiling so $\mathbf{t}+\mathbf{I}-\left(\mathbf{t}^{\prime}\right.$ $\left.+\mathbf{I}^{\prime}\right)=\mathbf{t}^{\prime \prime}+\mathbf{l}^{\prime \prime}$, where $\mathbf{t}^{\prime \prime} \in \mathbf{T}, \mathbf{l}^{\prime \prime} \in \mathbf{L}$. We have

$$
\mathbf{t} \varphi+h-\left(\mathbf{t}^{\prime} \varphi+h^{\prime}\right)=\mathbf{t}^{\prime \prime} \varphi+h^{\prime \prime} ; \quad h, h^{\prime}, h^{\prime \prime} \in H,
$$

with the application of homomorphism $\varphi$, which was to be proved. 
REMARKS. (1) Theorem 2.1 is known for certain sets $T$ in the following cases: $H=\{0\}$ in $[2,15] ; q_{1}=\cdots=q_{n}=1$ in $[8,10] ; H=\{0\}$ and $q_{1}=\cdots=q_{n}=1$ in $[6,7,10$ and 14].

(2) Theorem 2.1 was proved for general $T$ in the special case $H=\{0\}, q_{1}=\cdots=$ $q_{n}=1$ in [13].

We shall construct a vector set $\mathbf{L}$ for a system $\left(\mathcal{T}_{O}, \mathbf{L}\right), \mathbf{L} \subseteq \mathbf{X}$, in the special case when a basis for $G$ is a subset of $\left\{g_{1}, \ldots, g_{n}\right\}$.

Let $G$ be an abelian group expressible in the form (2.1), where $H=\left\{h_{1}, \ldots, h_{t}\right\}$. We shall assume that $g_{1}, \ldots, g_{s}, s<n$, is a basis for $G$, that is, $G$ is given by the relations $m_{1} g_{1}=0, \ldots, m_{s} g_{s}=0$. Thus

$$
\begin{cases}g_{v}=a_{v 1} g_{1}+\cdots+a_{v s} g_{s} ; & (s+1 \leqslant v \leqslant n), \\ h_{w}=b_{w s} g_{1}+\cdots+b_{w s} g_{s} ; & (1 \leqslant w \leqslant t) .\end{cases}
$$

Define $n+t$ vectors in $\mathbf{X}$ as follows:

$$
\left\{\begin{array}{l}
\mathbf{l}_{u}=\left(m_{u} / q_{u}\right) \mathbf{e}_{u} ; \quad(1 \leqslant u \leqslant s), \\
\mathbf{l}_{v}=\left(a_{v 1} / q_{1}\right) \mathbf{e}_{1}+\cdots+\left(a_{v s} / q_{s}\right) \mathbf{e}_{s}-\left(1 / q_{v}\right) \mathbf{e}_{v} ; \quad(s+1 \leqslant v \leqslant n), \\
\mathbf{l}_{n+w}=\left(b_{w 1} / q_{1}\right) \mathbf{e}_{1}+\cdots+\left(b_{w s} / q_{s}\right) \mathbf{e}_{s} ; \quad(1 \leqslant w \leqslant t),
\end{array}\right.
$$

and the following vector set

$$
\mathbf{L}:=\left\langle\mathbf{I}_{1}, \ldots, \mathbf{I}_{n}\right\rangle+\left\{\mathbf{I}_{n+1}, \ldots, \mathbf{I}_{n+t}\right\} .
$$

THEOREM 2.2. $H \varphi^{-1}=\mathbf{L}$.

Proof. We shall show that if $l \in \mathbf{L}$, then $l \in H \varphi^{-1}$, that is, $l \varphi \in H$. Assume that $\mathbf{I} \in \mathbf{L}$ so $\mathbf{I}=x_{1} \mathbf{I}_{1}+\cdots+x_{n} \mathbf{I}_{n}+\mathbf{I}_{n+w}$, where $x_{1}, \ldots, x_{n} \in Z ; 1 \leqslant w \leqslant t$. Thus $\mathbf{l} \varphi=$ $x_{1} I_{1} \varphi+\cdots+x_{n} I_{n} \varphi+I_{n+w} \varphi$. It is obvious that $I_{u} \varphi=0,1 \leqslant u \leqslant n$. Indeed

$$
\mathbf{l}_{u} \varphi=\left(m_{u} / q_{u}\right) \mathbf{e}_{u} \varphi=\left(m_{u} / q_{u}\right) q_{u} g_{u}=0 ; \quad(1 \leqslant u \leqslant s)
$$

and

$$
\begin{aligned}
\mathrm{l}_{v} \varphi & =\frac{a_{v 1}}{q_{1}} \mathbf{e}_{1} \varphi+\cdots+\frac{a_{v s}}{q_{s}} \mathbf{e}_{s} \varphi-\frac{1}{q_{v}} \mathbf{e}_{v} \varphi \\
& =\frac{a_{v 1}}{q_{1}} q_{1} g_{1}+\cdots+\frac{a_{v s}}{q_{s}} q_{s} g_{s}-\frac{1}{q_{v}} q_{v} g_{v} \\
& =a_{v 1} g_{1}+\cdots+a_{v s} g_{s}-g_{v}=0 ; \quad(s+1 \leqslant v \leqslant n) .
\end{aligned}
$$

Finally $I_{\varphi}=I_{n+w} \varphi$. It is obvious that $I_{n+w} \varphi \in H$. Indeed

$$
I_{n+w} \varphi=\frac{b_{w 1}}{q_{1}} \mathbf{e}_{1} \varphi+\cdots+\frac{b_{w s}}{q_{1}} \mathbf{e}_{s} \varphi=b_{w 1} g_{1}+\cdots+b_{w s} g_{s}=h_{w} \in H .
$$

Thus $\mathbf{L} \subseteq H \varphi^{-1}$, which was to be proved.

Now we shall show that $\mathrm{L} \supseteq H_{\varphi}{ }^{-1}$. In other words we shall show that if $\mathbf{x} \in \mathbf{X}$ and $\mathbf{x} \varphi \in H$, then $\mathbf{x} \in \mathbf{L}$.

Assume that $\mathbf{x} \varphi=h_{w}$. Since $\mathrm{I}_{n+w} \varphi=h_{w}$, we have $\left(\mathbf{x}-\mathrm{I}_{n+w}\right) \varphi=0$. Since $(\mathbf{x}-$ $\left.\mathbf{I}_{n+w}\right) \in \mathbf{X}$,

$$
\mathbf{x}-\mathbf{I}_{n+w}=\left(x_{1} / q_{1}\right) \mathbf{e}_{1}+\cdots+\left(x_{n} / q_{n}\right) \mathbf{e}_{n} ; \quad x_{1}, \ldots, x_{n} \in Z .
$$


By virtue of (2.6) and (2.7) it is clear that

$$
\mathbf{x}-\mathbf{I}_{n+w}+x_{s+1} \mathbf{I}_{s+1}+\cdots+x_{n} \mathbf{I}_{n}=\left(x_{1}^{\prime} / q_{1}\right) \mathbf{e}_{1}+\cdots+\left(x_{s}^{\prime} / q_{s}\right) \mathbf{e}_{s}
$$

for some integers $x_{1}^{\prime}, \ldots, x_{s}^{\prime}$. Let $u_{i}$ and $r_{i}$ be integers with the property that $x_{i}^{\prime}=u_{i} m_{i}+r_{i}, 0 \leqslant r_{i}<m_{i}, 1 \leqslant i \leqslant s$. Thus

$$
\mathbf{x}-\mathbf{I}_{n+w}+x_{s+1} \mathbf{l}_{s+1}+\cdots+x_{n} \mathbf{l}_{n}=u_{1} \mathbf{l}_{1}+\cdots+u_{s} \mathbf{l}_{s}+\frac{r_{1}}{q_{1}} \mathbf{e}_{1}+\cdots+\frac{r_{s}}{q_{s}} \mathbf{e}_{s} .
$$

Applying the homomorphism $\varphi$, we find that $0=r_{1} g_{1}+\cdots+r_{s} g_{s}$. Since $\left\{g_{1}, \ldots, g_{s}\right\}$ is a basis for $G$ and $0 \leqslant r_{i}<m_{i}, 1 \leqslant i \leqslant s$, it follows that $r_{1}=\cdots=r_{s}=0$. Returning to (2.8), we conclude that $\left(\mathbf{x}-\mathbf{I}_{n+w}\right) \in \mathbf{L}$, which was to be proved.

The following theorem serves as a basis for our construction of nonlattice tiling $\left(\sigma_{O}, \mathbf{L}\right)$.

THEOREM 2.3. Assume that the abelian group $G$ is expressible in the form (2.1), where $H=\{0\},\left(q_{1}, \ldots, q_{n}\right) \neq(1, \ldots, 1)$ and $\left\langle q_{1} g_{1}, \ldots, q_{n} g_{n}\right\rangle=G$. Then $G$ is expressible in the form

$$
G=T\left(g_{1}^{\prime}, \ldots, g_{n}^{\prime}\right)+H^{\prime},
$$

where $\left\langle g_{1}^{\prime}, \ldots, g_{n}^{\prime}\right\rangle=G$ and $H^{\prime} \Varangle G$.

Proof. Assume that $q_{1}>1, \ldots, q_{s}>1, q_{s+1}=\cdots=q_{n}=1(s \geqslant 1)$. Let $g_{1}^{\prime}=$ $q_{1} g_{1}, \ldots, g_{n}^{\prime}=q_{n} g_{n}$ and

$$
H^{\prime}=\left\{0, g_{1}, \ldots,\left(q_{1}-1\right) g_{1}\right\}+\cdots+\left\{0, g_{s}, \ldots,\left(q_{s}-1\right) g_{s}\right\}
$$

So $G$ is expressible in the form (2.9) and $\left\langle g_{1}^{\prime}, \ldots, g_{n}^{\prime}\right\rangle=G$.

If $H^{\prime} \subseteq G$ were a subgroup of $G$, then it would follow that

$$
\left(\left\{0, g_{1}, \ldots,\left(q_{1}-1\right) g_{1}\right\}+\cdots+\left\{0, g_{s}, \ldots,\left(q_{s}-1\right) g_{s}\right\}\right) \triangleleft G
$$

and therefore, according to Hajós' theorem on the factorization of finite abelian groups (see [2]), at least one set $\left\{0, g_{i}, \ldots,\left(q_{i}-1\right) g_{i}\right\}$ would be a subgroup of $G$. In other words, at least one $q_{i} g_{i}=0$. Finally, since (2.1) is a factorization of $G$ and therefore $0 \neq q_{i} g_{i}$, it follows that $H^{\prime}$ is not a subgroup of $G$.

3. The algebra of crosses. Let $G, G^{\prime}, G^{\prime \prime}$ be finite abelian groups, $\Delta^{\prime} \subseteq G^{\prime}$, $\Delta^{\prime \prime} \subseteq G^{\prime \prime}$ and let

$$
\{0\} \rightarrow G \stackrel{\alpha}{\rightarrow} \Delta^{\prime} \stackrel{\beta}{\rightarrow} \Delta^{\prime \prime} \rightarrow\{0\}
$$

be an exact sequence. (That is, $\alpha$ is an isomorphism, $\beta$ is a homomorphism, $\operatorname{Ker} \beta=\operatorname{Im} \alpha$, and $\operatorname{Im} \beta=\Delta^{\prime \prime}$.) In this case if $A$ is a subset of $\Delta^{\prime}$ with the property that the restriction of $\beta$ to $A$ is a bijection between $A$ and $\Delta^{\prime \prime}$, then $\Delta^{\prime}=A+G \alpha$ is a factorization of $\Delta^{\prime}$.

Conversely, let $\Delta^{\prime}=A+B$ be a factorization of $\Delta^{\prime}$ such that $B$ is a subgroup of $G^{\prime}$. If $\alpha$ is the inclusion mapping and $\beta$ is the restriction to $\Delta^{\prime}$ of the natural 
homomorphism from $G^{\prime}$ to $G^{\prime} / B$, then

$$
\{0\} \rightarrow B \stackrel{\alpha}{\rightarrow} \Delta^{\prime} \stackrel{\beta}{\rightarrow} A \rightarrow\{0\}
$$

is an exact sequence.

Let $s_{1} \ldots, s_{k}$ be distinct nonzero integers and let $S_{n, k}$ be the following subset of $Z^{n}$ :

$$
S_{n, k}:=\left\{(0, \ldots, 0),(i, 0, \ldots, 0), \ldots,(0, \ldots, 0, i): i \in\left\{s_{1}, \ldots, s_{k}\right\}\right\}
$$

THEOREM 3.1. Assume that $s_{1}, \ldots, s_{k}$ are relatively prime to $|G|$ and that

$$
\{0\} \rightarrow G \stackrel{\alpha}{\rightarrow} \Delta^{\prime} \stackrel{\beta}{\rightarrow} \Delta^{\prime \prime} \rightarrow\{0\}
$$

is an exact sequence. Furthermore, assume that $G$ and $\Delta^{\prime \prime}$ are expressible in the form $G=S_{r, k}\left(g_{1}, \ldots, g_{r}\right), \Delta^{\prime \prime}=S_{t, k}\left(\delta_{1}^{\prime \prime}, \ldots, \delta_{t}^{\prime \prime}\right)$. For each $j, 1 \leqslant j \leqslant t$, select $\delta_{j} \in \Delta^{\prime}$ such that $\delta, \beta=\delta_{j}^{\prime \prime}$. Then $\Delta^{\prime}$ is expressible in the form $\Delta^{\prime}=S_{v, k}\left(\delta_{1}^{\prime}, \ldots, \delta_{v}^{\prime}\right)$, where

$$
\left(\delta_{1}^{\prime}, \ldots, \delta_{v}^{\prime}\right)=\left\{g_{1} \alpha, \delta_{j}+g \alpha: 1 \leqslant i \leqslant r, 1 \leqslant j \leqslant t, g \in G\right\} .
$$

Proof. This statement was proved by W. Hamaker and S. K. Stein in [5, p. 322] with the assumption that $\Delta^{\prime}$ is a group. The reader can readily verify that this assumption can be removed.

THEOREM 3.2. Assume that $s_{1}, \ldots, s_{k}$ are relatively prime to $|G|$. Then $G$ is expressible in the form $G=S_{r, k}\left(g_{1}, \ldots, g_{r}\right)$ if and only if for each prime divisor $p$ of $|G|$ the cyclic group $C_{p}$ of order $p$ is expressible in the form $C_{p}=S_{t, k}\left(c_{1}, \ldots, c_{t}\right)$.

For a proof see $[5$, p. 324].

THEOREM 3.3. Assume that $s_{1}, \ldots, s_{k}$ are relatively prime to $|G|$. Assume that $G$ is given by $m_{1} g_{1}=0, \ldots, m_{s} g_{s}=0$ and that $G$ is expressible in the form $G=$ $S_{r, k}\left(g_{1}^{\prime}, \ldots, g_{r}^{\prime}\right)$. Then $G$ is expressible in the form $G=S_{r, k}\left(g_{1}^{\prime \prime}, \ldots, g_{r}^{\prime \prime}\right)$ such that $\left\{g_{1}, \ldots, g_{s}\right\} \subseteq\left\{g_{1}^{\prime \prime}, \ldots, g_{r}^{\prime \prime}\right\}$.

Proof. If $\alpha$ is the inclusion mapping and $\beta$ is the natural homomorphism between $\left\langle g_{1}, \ldots, g_{i+1}\right\rangle$ and $\left\langle g_{1}, \ldots, g_{i+1}\right\rangle /\left\langle g_{i+1}\right\rangle$, then

$$
\{0\} \rightarrow\left\langle g_{i+1}\right\rangle \stackrel{\alpha}{\rightarrow}\left\langle g_{1}, \ldots, g_{i+1}\right\rangle \stackrel{\beta}{\rightarrow}\left\langle g_{1}, \ldots, g_{1}\right\rangle \rightarrow\{0\}
$$

is an exact sequence. On the basis of the assumption that the statement of the theorem is true in the case $s=1$ it follows that the statement of the theorem is true for every s. (Use Theorem 3.1 and induction on $i$.)

We shall restrict our consideration to the case $s=1$. Let $g$ be a generator of the cyclic group $G$. By assumption, there is an integer $s_{j}$ and an element $g_{t}^{\prime}$ such that $s_{j} g_{t}^{\prime}=g$. Since $s_{j}$ is relatively prime to $G$, the homomorphism $\psi: G \rightarrow G$ defined by $h \psi=s_{j} h, h \in G$, is an automorphism. Thus $G=S_{r . k}\left(g_{1}^{\prime} \psi, \ldots, g_{r}^{\prime} \psi\right)$ and, since $g_{t}^{\prime} \psi=g$, the theorem is proved. 
THEOREM 3.4. Assume that $s_{1}, \ldots, s_{k}$ are relatively prime to $|G|$ and that $G$ is expressible in the form $G=S_{n, k}\left(g_{1}, \ldots, g_{n}\right)$, where $n \geqslant 2$. If $G$ is not a prime number, then there is an abelian group $\Gamma$ which is expressible in the form

$$
\begin{aligned}
\Gamma= & S_{n, k}\left(q_{1} \gamma_{1}, \ldots, q_{n} \gamma_{n}\right) \\
& +\left\{0, \gamma_{1}, \ldots,\left(q_{1}-1\right) \gamma_{1}\right\}+\cdots+\left\{0, \gamma_{n}, \ldots,\left(q_{n}-1\right) \gamma_{n}\right\},
\end{aligned}
$$

where $S_{n, k}\left(q_{1} \gamma_{1}, \ldots, q_{n} \gamma_{n}\right) \Varangle \Gamma,\left\langle q_{1} \gamma_{1}, \ldots, q_{n} \gamma_{n}\right\rangle=\Gamma$.

Proof. $|G|$ is not a prime number so $|G|=u \cdot v, u>1, v>1$. The cyclic group $\Gamma$ is defined by $2 u v \gamma=0$. If $\alpha$ is the inclusion mapping from $\langle v \gamma\rangle$ to $\langle\gamma\rangle$ and $\beta$ is the homomorphism $\langle\gamma\rangle \rightarrow\langle 2 u \gamma\rangle$ given by $\gamma \beta=2 u \gamma$, then

$$
\{0\} \rightarrow\langle v \gamma\rangle \stackrel{\alpha}{\rightarrow}\langle\gamma\rangle \stackrel{\beta}{\rightarrow}\langle 2 u \gamma\rangle \rightarrow\{0\}
$$

is an exact sequence. According to Theorem 3.2, $\langle 2 u \gamma\rangle$ is expressible in the form $\langle 2 u \gamma\rangle=S_{t, k}\left(a_{1} 2 u \gamma, \ldots, a_{t} 2 u \gamma\right), a_{1}, \ldots, a_{t} \in Z$. According to Theorem 3.3 we may assume that $a_{1}=1$. Define the set $A_{1} \subseteq\langle\gamma\rangle$ by $A_{1}=S_{t, k}\left(a_{1} \gamma, \ldots, a_{t} \gamma\right)$. It is clear that $\langle 2 u \gamma\rangle$ is isomorphic to $\langle\gamma\rangle /\langle v \gamma\rangle=\{i \gamma+\langle v \gamma\rangle: 0 \leqslant i\langle v\}$. Furthermore, it is clear that the restriction of $\beta$ to $A_{1}$ is a bijection between $A_{1}$ and $\langle 2 u \gamma\rangle$. Thus $\langle\gamma\rangle=A_{1}+\langle v \gamma\rangle$ is a factorization of $\langle\gamma\rangle$.

There is an exact sequence

$$
\{0\} \rightarrow\langle 2 v \gamma\rangle \rightarrow\langle v \gamma\rangle \rightarrow\langle u v \gamma\rangle \rightarrow\{0\} .
$$

Define the set $A_{2} \subseteq\langle v \gamma\rangle$ by $A_{2}=\{0, v \gamma\}$. Then $\langle v \gamma\rangle=A_{2}+\langle 2 v \gamma\rangle$ is a factorization of $\langle v \gamma\rangle$.

Finally, $\langle\gamma\rangle=A_{1}+A_{2}+\langle 2 v \gamma\rangle$ is a factorization of $\langle\gamma\rangle$. According to Theorem $3.2,\langle 2 v \gamma\rangle$ is expressible in the form $\langle 2 v \gamma\rangle=S_{w, k}\left(b_{1}, \ldots, b_{w}\right)$. According to Theorem 3.3 we may assume that $b_{1}=2 v \gamma$. Note that there is an exact sequence

$$
\{0\} \rightarrow\langle 2 v \gamma\rangle \rightarrow A_{1}+\langle 2 v \gamma\rangle \rightarrow A_{1} \rightarrow\{0\} .
$$

According to Theorem 3.1 we have $A_{1}+\langle 2 v \gamma\rangle=S_{n, k}\left(d_{1}, \ldots, d_{n}\right)$, where $d_{1}=2 v \gamma$, $d_{w+1}=\gamma$. If $q_{1}=2, q_{2}=\cdots=q_{n}=1, \gamma_{1}=v \gamma, \gamma_{2}=d_{2}, \ldots, \gamma_{n}=d_{n}$, then $\Gamma$ is expressible in the form (3.1). It is clear from the definitions of $q_{1}, \ldots, q_{n}, \gamma_{1}, \ldots, \gamma_{n}$ that $\left\langle q_{1} \gamma_{1}, \ldots, q_{n} \gamma_{n}\right\rangle=\langle\gamma\rangle=\Gamma$.

We shall show that $A_{1}+\langle 2 v \gamma\rangle=S_{n, k}\left(q_{1} \gamma_{1}, \ldots, q_{n} \gamma_{n}\right)$ is not a subgroup of $\Gamma$. Assume that $\left(A_{1}+\langle 2 v \gamma\rangle\right) \triangleleft \Gamma$. Since $\gamma \in\left(A_{1}+\langle 2 v \gamma\rangle\right),\langle\gamma\rangle \triangleleft\left(A_{1}+\langle 2 v \gamma\rangle\right)$, but $2 u v=|\gamma|>\left|A_{1}+\langle 2 v \gamma\rangle\right|=u v$.

4. Tiling by semicrosses and full crosses. By considering a special type of set $T$, we shall examine the problem of tiling Euclidean space by crosses and semicrosses.

Let $K^{*}(n, k) \subseteq Z^{n}$ denote the set

$$
K^{*}(n, k):=\{(0, \ldots, 0),(i, 0, \ldots, 0), \ldots,(0, \ldots, 0, i): 1 \leqslant i \leqslant k\}
$$

and let

$$
\begin{aligned}
\mathbf{K}^{*}(n, k) & :=\left\{i \mathbf{e}_{j}: 0 \leqslant i \leqslant k, 1 \leqslant j \leqslant n\right\} \quad \text { and } \\
\mathfrak{K}_{O}^{*}(n, k) & :=\left\{\bigodot_{P}: \overrightarrow{O P} \in \mathbf{K}^{*}(n, k)\right\} .
\end{aligned}
$$


The set of cubes $\mathcal{K}_{O}^{*}(n, k)$ consists of $k n+1$ cubes formed of $n$ arms of length $k$ attached at a central cube. In a similar manner we define the sets $K(n, k), \mathbf{K}(n, k)$, $\mathcal{K}_{O}(n, k)$ :

$$
\begin{aligned}
& K(n, k):=\{(0, \ldots, 0),(i, 0, \ldots, 0), \ldots,(0, \ldots, 0, i): 1 \leqslant|i| \leqslant k\}, \\
& \mathbf{K}(n, k):=\left\{i \mathbf{e}_{j}: 0 \leqslant|i| \leqslant k, 1 \leqslant j \leqslant n\right\}, \\
& \mathscr{K}_{O}(n, k):=\left\{\bigodot_{P}: \overrightarrow{O P} \in \mathbf{K}(n, k)\right\} .
\end{aligned}
$$

The set of cubes $\mathcal{K}_{o}(n, k)$, which consists of $2 k n+1$ cubes formed of $2 n$ arms of length $k$ meeting at a central cube, is called a cross.

It is obvious that if $s_{1}=1, s_{2}=2, \ldots, s_{k}=k$, then $S_{n, k}=K^{*}(n, k)$ and if $s_{1}=1$, $s_{2}=2, \ldots, s_{k}=k, s_{k+1}=-1, s_{k+2}=-2, \ldots, s_{2 k}=-k$, then $S_{n, 2 k}=K(n, k)$.

THEOREM 4.1. If there is an integer lattice tiling $\left(\mathcal{K}_{O}^{*}(n, k), \mathbf{L}\right), \mathbf{L} \triangleleft \mathbf{M}$, if $k n+1$ is not a prime, and if $p>k$ for every prime divisor $p$ of $k n+1$, then there are

(1) a noninteger lattice tiling $\left(\mathcal{K}_{o}^{*}(n, k), \mathbf{L}^{\prime}\right), \mathbf{L}^{\prime} \nsubseteq \mathbf{M}, \mathbf{L}^{\prime} \triangleleft \mathbf{X}$, and

(2) an integer nonlattice tiling $\left(\mathcal{K}_{O}^{*}(n, k), \mathbf{L}^{\prime \prime}\right), \mathbf{L}^{\prime \prime} \subseteq \mathbf{M}, \mathbf{L}^{\prime \prime} \Varangle \mathbf{M}$.

Proof. (1) follows immediately from Theorems 2.1 and 3.4. (2) follows from Theorems 2.1, 2.3 and 3.4.

THEOREM 4.2. If there is an integer lattice tiling $\left(\mathcal{K}_{O}(n, k), \mathbf{L}\right), \mathbf{L} \triangleleft \mathbf{M}$, such that $2 k n+1$ is not a prime and $p>k$ for every prime divisor $p$ of $2 k n+1$, then there are

(1) a noninteger lattice tiling $\left(\mathcal{K}_{o}(n, k), \mathbf{L}^{\prime}\right), \mathbf{L}^{\prime} \nsubseteq \mathbf{M}, \mathbf{L}^{\prime} \triangleleft \mathbf{X}$, and

(2) an integer nonlattice tiling $\left(\mathscr{K}_{O}(n, k), \mathbf{L}^{\prime \prime}\right), \mathbf{L}^{\prime \prime} \subset \mathbf{M}, \mathbf{L}^{\prime \prime} \phi \mathbf{M}$.

The proof is similar to that of the preceding theorem.

The next theorem presents a contrast to the last two theorems.

THEOREM 4.3. For any lattice tiling $\left(\sigma_{O}, \mathbf{L}\right)$, where $\left|\mathcal{T}_{O}\right|$ is a prime number, $\mathbf{L} \subseteq \mathbf{M}$.

Proof. This was proved for special $\mathcal{T}_{O}$ in [15] with the aid of Rèdei's theorem on the factorization of abelian groups into sets with a prime number of elements. The reader can readily verify that this proof holds for a general $\widetilde{T}_{O}$.

5. An example. We now illustrate our theorems by considering a cross with arms of length two in 55-space. Now $4 \cdot 55+1=221=13 \cdot 17$ and $13>2,17>2$. The group $C$ is defined by $13 c=0$ and the group $C^{\prime}$ is defined by $17 c^{\prime}=0$. It is clear that

$$
C=K(3,2)(c, 4 c, 3 c), \quad C^{\prime}=K(4,2)\left(c^{\prime}, 4 c^{\prime}, 3 c^{\prime}, 12 c^{\prime}\right) .
$$

So the abelian group $G$ of order 221 is expressible in the form $G=$ $K(55,2)\left(g_{1}, \ldots, g_{55}\right)$. Thus there is an integer lattice tiling $\left(\mathscr{K}_{O}(55,2), \mathbf{L}\right), \mathbf{L} \triangleleft \mathbf{M}$. Now we shall construct a noninteger lattice tiling

$\left(\mathscr{K}_{o}(55,2), \mathbf{L}^{\prime}\right), \mathbf{L}^{\prime} \nsubseteq \mathbf{M}, \mathbf{L}^{\prime} \triangleleft \mathbf{X}$, and an integer nonlattice tiling $\left(\mathscr{K}_{O}(55,2), \mathbf{L}^{\prime \prime}\right)$, $\mathbf{L}^{\prime \prime} \subseteq \mathbf{M}, \mathbf{L}^{\prime \prime} \phi \mathbf{M}$.

Consider the group $\Gamma=\langle\gamma\rangle$ defined by $2 \cdot 221 \gamma=0$. There is an exact sequence

$$
\{0\} \rightarrow\langle 17 \gamma\rangle \rightarrow\langle\gamma\rangle \rightarrow\langle 26 \gamma\rangle \rightarrow\{0\} \text {. }
$$


Thus $\langle\gamma\rangle=A_{1}+\langle 17 \gamma\rangle$, where $A_{1}$ is $K(4,2)(\gamma, 4 \gamma, 3 \gamma, 12 \gamma)=\{0, \gamma, 2 \gamma,-\gamma,-2 \gamma$, $4 \gamma, 8 \gamma,-4 \gamma,-8 \gamma, 3 \gamma, 6 \gamma,-3 \gamma,-6 \gamma, 12 \gamma, 24 \gamma,-12 \gamma,-24 \gamma\}$. There is an exact sequence

$$
\{0\} \rightarrow\langle 34 \gamma\rangle \rightarrow\langle 17 \gamma\rangle \rightarrow\langle 221 \gamma\rangle \rightarrow\{0\}
$$

so $\langle 17 \gamma\rangle=A_{2}+\langle 34 \gamma\rangle$, where $A_{2}=\{0,17 \gamma\}$.

Finally

$$
\begin{aligned}
& \langle\gamma\rangle=A_{1}+A_{2}+\langle 34 \gamma\rangle=\left(A_{1}+\langle 34 \gamma\rangle\right)+A_{2} \\
& =K(55,2)(34 \gamma, 4 \cdot 34 \gamma, 3 \cdot 34 \gamma, \gamma, \gamma+34 \gamma, \gamma+2 \cdot 34 \gamma, \ldots \text {, } \\
& \gamma+12 \cdot 34 \gamma, 4 \gamma, 4 \gamma+34 \gamma, 4 \gamma+2 \cdot 34 \gamma, \ldots, \\
& 4 \gamma+12 \cdot 34 \gamma, 3 \gamma, 3 \gamma+34 \gamma, 3 \gamma+2 \cdot 34 \gamma, \ldots \text {, } \\
& 3 \gamma+12 \cdot 34 \gamma, 12 \gamma, 12 \gamma+34 \gamma, 12 \gamma+2 \cdot 34 \gamma, \ldots \text {, } \\
& 12 \gamma+12 \cdot 34 \gamma)+\{0,17 \gamma\} \text {. } \\
& \text { If } q_{1}=2, q_{2}=\cdots=q_{55}=1 ; \gamma_{1}=17 \gamma, \gamma_{2}=136 \gamma, \gamma_{3}=102 \gamma \text {, } \\
& \gamma_{4}=\gamma, \quad \gamma_{5}=35 \gamma \quad, \ldots, \quad \gamma_{16}=409 \gamma, \\
& \gamma_{17}=4 \gamma, \quad \gamma_{18}=38 \gamma \quad, \ldots, \quad \gamma_{29}=412 \gamma, \\
& \gamma_{30}=3 \gamma, \quad \gamma_{31}=37 \gamma \quad, \ldots, \quad \gamma_{42}=411 \gamma, \\
& \gamma_{43}=12 \gamma, \quad \gamma_{44}=46 \gamma \quad, \ldots, \quad \gamma_{55}=420 \gamma \text {, }
\end{aligned}
$$

then $\Gamma$ is expressible in the form

$$
\begin{aligned}
\Gamma= & K(55,2)\left(q_{1} \gamma_{1}, \ldots, q_{55} \gamma_{55}\right) \\
& +\left\{0, \gamma_{1}, \ldots,\left(q_{1}-1\right) \gamma_{1}\right\}+\cdots+\left\{0, \gamma_{55}, \ldots,\left(q_{55}-1\right) \gamma_{55}\right\} .
\end{aligned}
$$

According to Theorem 2.1 there is a noninteger lattice tiling $\left(\mathscr{K}_{o}(55,2), \mathbf{L}^{\prime}\right)$. According to Theorem 2.2 the vectors

$$
\begin{aligned}
\mathbf{l}_{1}^{\prime} & =-\frac{1}{2} \mathbf{e}_{1}+17 \mathbf{e}_{4}, \mathbf{l}_{2}^{\prime}=-\mathbf{e}_{2}+136 \mathbf{e}_{4}, \mathbf{l}_{3}^{\prime}=-\mathbf{e}_{3}+102 \mathbf{e}_{4}, \mathbf{l}_{4}^{\prime}=442 \mathbf{e}_{4}, \\
\mathbf{l}_{5}^{\prime} & =35 \mathbf{e}_{4}-\mathbf{e}_{5}, \ldots, \mathbf{l}_{16}^{\prime}=409 \mathbf{e}_{4}-\mathbf{e}_{16}, \\
\mathbf{l}_{17}^{\prime} & =4 \mathbf{e}_{4}-\mathbf{e}_{17}, \mathbf{l}_{18}^{\prime}=38 \mathbf{e}_{4}-\mathbf{e}_{18}, \ldots, l_{29}^{\prime}=412 \mathbf{e}_{4}-\mathbf{e}_{29}, \\
\mathbf{l}_{30}^{\prime} & =3 \mathbf{e}_{4}-\mathbf{e}_{30}, \mathbf{l}_{31}^{\prime}=37 \mathbf{e}_{4}-\mathbf{e}_{31}, \ldots, \mathbf{l}_{42}^{\prime}=411 \mathbf{e}_{4}-\mathbf{e}_{42}, \\
\mathbf{l}_{43}^{\prime} & =12 \mathbf{e}_{4}-\mathbf{e}_{43}, \mathbf{l}_{44}^{\prime}=46 \mathbf{e}_{4}-\mathbf{e}_{44}, \ldots, l_{55}^{\prime}=420 \mathbf{e}_{4}-\mathbf{e}_{55}
\end{aligned}
$$

span the lattice $\mathbf{L}^{\prime}$, that is, $\mathbf{L}^{\prime}=\left\langle\mathbf{l}_{1}^{\prime}, \ldots, \mathbf{l}_{55}^{\prime}\right\rangle$.

$\Gamma$ is expressible in the form

$$
\Gamma=K(55,2)\left(\bar{\gamma}_{1}, \ldots, \bar{\gamma}_{55}\right)+H,
$$

where $\bar{\gamma}_{1}=34 \gamma, \bar{\gamma}_{2}=\gamma_{2}, \ldots, \bar{\gamma}_{55}=\gamma_{55} ; H=\{0,17 \gamma\} ; H$ is not a subgroup of $\Gamma$; $\left\langle\bar{\gamma}_{1}, \ldots, \bar{\gamma}_{55}\right\rangle=\Gamma$. Thus according to Theorem 2.1 there is an integer nonlattice tiling $\left(\mathcal{K}_{o}(55,2), \mathbf{L}^{\prime \prime}\right)$. If $I_{1}^{\prime \prime}=-\mathbf{e}_{1}+34 \mathbf{e}_{4}, l_{2}^{\prime \prime}=l_{2}^{\prime}, \ldots, l_{55}^{\prime \prime}=I_{55}, l_{56}^{\prime \prime}=0, l_{57}^{\prime \prime}=17 \mathbf{e}_{4}$, then according to Theorem $2.2, \mathbf{L}^{\prime \prime}=\left\langle\mathbf{l}_{1}^{\prime \prime}, \ldots, \mathbf{l}_{55}^{\prime \prime}\right\rangle+\left\{\mathbf{l}_{56}^{\prime \prime}, \mathbf{l}_{57}^{\prime \prime}\right\}$.

The author would like to thank the referee for his valuable suggestions and a short proof of Theorem 3.3. 


\section{REFERENCES}

1. H. Everett and D. Hickerson, Packing and covering by translates of certain nonconvex bodies, Proc. Amer. Math. Soc. 75 (1979), 87-91.

2. G. Hajos, Über einfache und mehrfache Bedeckung des n-dimensionalen Raumes mit einem Würfelgitter, Math. Z. 47 (1942), 427-467.

3. Sur la factorisation des groupes abèlians, Časopis Pěst. Mat. Fys. 74 (1949), 157- 162.

4. W. Hamaker, Factoring groups and tiling space, Aequationes Math. 9(1973), 145-149.

5. W. Hamaker and S. K. Stein, Splitting groups by integers, Proc. Amer. Math. Soc. 46 (1974). $322-324$

6. E. Molnár, Sui mosaici dello spacio di dimensione n, Atti Accad. Naz. Lincei Rend. Cl. Sci. Fis. Mat. Natur. 51 (1971), 177-185.

7. S. K. Stein, Factoring by subsets, Pacific J. Math. 22 (1967), 523-541.

8. A symmetric star body that tiles hut not as a lattice, Proc. Amer. Math. Soc. 36 (1972), $543-548$.

9. Tiling space by congruent polyhedra, Bull. Amer. Math. Soc. 80 (1974), 819-820.

10. Algebraic tiling. Amer. Math. Monthly 81 (1974), 445-462.

11. S. Szabó, A mode of decomposition for finite abelian groups, K. Marx Univ. of Economics, Dept. of Math., Budapest, 1979.

12. On decomposing finite abelian groups, Acta Math. Acad. Sci. Hungar. 36 (1980), 105-114.

13. Véges abel-csoportok és n-dimenziós mozaikok, Mat. Lapok 28 (1977-1980), 305-318.

14. _. О связи конечных абелевых грушп и решетчатых мозанх в $n$-мерном эвклидовом пространстве, Ann. Univ. Sci. Budapest. Eötvös Sect. Math. 24 (1981), 29-43.

15. On mosaics consisting of multidimensional crosses, Acta Math. Acad. Sci. Hungar. 38 (1981), $191-203$.

Department of Civil Engineering Mathematics, TeChnical University, Budapest, 1111 BudaPEST, STOCZEK U.2., HUNGARY 